\title{
A Competitive Mean-Squared Error Approach to Beamforming
}

\author{
Yonina C. Eldar, Member, IEEE, Arye Nehorai, Fellow, IEEE, and Patricio S. La Rosa
}

\begin{abstract}
We treat the problem of beamforming for signal estimation where the goal is to estimate a signal amplitude from a set of array observations. Conventional beamforming methods typically aim at maximizing the signal-to-interference-plus-noise ratio (SINR). However, this does not guarantee a small mean-squared error (MSE), so that on average the resulting signal estimate can be far from the true signal. Here, we consider strategies that attempt to minimize the MSE between the estimated and unknown signal waveforms. The methods we suggest all maximize the SINR but at the same time are designed to have good MSE performance. Since the MSE depends on the signal power, which is unknown, we develop competitive beamforming approaches that minimize a robust MSE measure. Two design strategies are proposed: minimax MSE and minimax regret. We demonstrate through numerical examples that the suggested minimax beamformers can outperform several existing standard and robust methods, over a wide range of signal-to-noise ratio (SNR) values. Finally, we apply our techniques to subband beamforming and illustrate their advantage in estimating a wideband signal.
\end{abstract}

Index Terms-Beamforming, minimax mean-squared error, minimax regret, robust beamforming, subband beamforming.

\section{INTRODUCTION}

B EAMFORMING is a classical method of processing temporal sensor array measurements for signal estimation, interference cancellation, source direction, and spectrum estimation. It has ubiquitously been applied in areas such as radar, sonar, wireless communications, speech processing, and medical imaging (see, e.g., [1]-[4] and references therein).

Conventional approaches for designing data dependent beamformers typically attempt to maximize the signal-to-interferenceplus-noise ratio (SINR). Maximizing the SINR requires knowledge of the interference-plus-noise covariance matrix and the array steering vector. Since this covariance is usually unknown, it is often replaced by the sample covariance of the measurements, resulting in performance deterioration at high signal-tonoise ratio (SNR) when the signal is present in the training data.

Manuscript received January 18, 2005; revised January 29, 2007. The associate editor coordinating the review of this manuscript and approving it for publication was Dr. Nicholas D. Sidiropoulos. The work of Y. Eldar was supported by the Israel Science Foundation under Grant No. 536/04 and by the GlasbergKlein Research Fund. The work of A. Nehorai was supported by the Air Force Office of Scientific Research Grants F49620-02-1-0339, FA9550-05-1-0018, and the National Science Foundation Grants CCR-0105334 and CCR-0330342.

Y. C. Eldar is with the Department of Electrical Engineering, TechnionIsrael Institute of Technology, Haifa, Israel 32000 (e-mail: yonina@ ee.technion. ac.il).

A. Nehorai and P. S. La Rosa are with the Department of Electrical and Systems Engineering, Washington University in St. Louis, St. Louis, MO 63130 USA (e-mail: nehorai@ese.wustl.edu).

Digital Object Identifier 10.1109/TSP.2007.897883
Some beamforming techniques are designed to mitigate this effect [5]-[8], whereas others are developed to also overcome uncertainty in the steering vector [9]-[13], [14]. Here, we assume that the steering vector is known exactly and our aim is to design a beamformer for signal estimation.

Despite the fact that the SINR has been used as a performance measure and as a design criterion in many beamforming approaches, maximizing SINR may not guarantee a good estimate of the signal. In an estimation context, where our goal is to design a beamformer in order to obtain an estimate of the signal amplitude that is close to its true value, it would make more sense to choose the weights to minimize an objective that is related to the estimation error, i.e., the difference between the true signal and its estimate, rather than the SINR. Furthermore, it may be more informative to consider the estimation error as a performance measure in comparing different beamforming methods.

If the signal power is known, then a minimum mean-squared error (MMSE) beamformer can be designed. The resulting beamformer can be expressed as a power-dependent constant which multiplies a fixed weight vector that is optimal in an SINR sense. Since the SINR is insensitive to scaling, the MMSE approach also maximizes the SINR. If the scaling is fixed, then the choice of scaling does not affect the signal waveform, but only its magnitude. In some applications, the actual magnitude value may be of importance. This is particularly true in the context of subband beamforming [15]-[20] which gained interest in recent years, due to its ability to reduce the complexity of conventional broadband strategies. In this context, beamforming is performed independently in decimated frequency bands, and the outputs of the channels are combined. Since different scaling coefficients are used in each channel, the MMSE strategy will generally result in a signal whose waveform is different than that resulting from an SINR-based method. Thus, a good choice of scaling factor can significantly impact the estimated waveform.

Typically, the signal power is unknown, and the MMSE beamformer cannot be realized. In such cases, other design criteria are needed to choose the scaling factor. A common approach is to select the scaling such that the signal is undistorted, which is equivalent to minimizing the mean-squared error (MSE) under the constrain that the beamformer is unbiased. This leads to the well known minimum variance distortionless response (MVDR) beamformer. However, as we show both analytically and in simulations, despite the fact that the MVDR method is optimal in an MSE sense among unbiased techniques, it often results in a large estimation error. An alternative strategy is to estimate the signal power from the data and use the power estimate in conjunction with the MMSE beamformer. 
This approach is closely related to the blind minimax-MSE techniques, recently developed in [21]. Based on the results of [21], it can be shown that if the signal power is properly estimated and the noise covariance is known, then this method can improve the MSE over the MVDR strategy. However, as we demonstrate via simulations in Section $\mathrm{V}$, in the typical scenario where the covariance is unknown, the performance using this method deteriorates considerably.

In order to develop a beamformer with good MSE performance when the signal power and noise covariance are unknown, in this paper we propose two design strategies that exploit prior knowledge on the signal power in the form of upper and lower bounds. Both methods optimize a worst-case MSE criterion over the feasible signal region. The advantage of these techniques is two-fold: First, they allow to explicitly incorporate prior knowledge on the signal power, which in many contexts is available, and in that way improve the MSE performance over other methods. Second, in settings where no prior knowledge is available, the power bounds can be easily estimated from the data, thus leading to practical beamforming techniques. Indeed, we demonstrate via simulations that using the estimated bounds in conjunction with the proposed strategies leads to improved MSE over previous beamformers.

The criteria we choose are based on recent ideas developed in the context of estimation in linear models [22]-[24]. In the first method, we minimize the worst-case MSE over all signals whose magnitude (or variance, in the zero-mean stochastic signal case) is bounded by a constant. In the second approach, we minimize the worst-case regret [23], [25], [26] over all bounded signals, where the regret is defined as the difference between the MSE of the beamformer output in the presence of uncertainties, and the smallest attainable MSE when the power is exactly known. This strategy considers both an upper and a lower bound on the signal magnitude. To illustrate the advantage of our methods we present several numerical examples comparing our beamformers with conventional SINR-based strategies, scaled to account for the MSE, and several recently proposed robust approaches [10]-[12]. We also apply our techniques to subband beamforming and illustrate their advantage in estimating a wideband signal.

The theoretical ideas developed here have also been reported in [13]. However, here we specifically address the practical aspects of the methods i.e., their performance in comparison with scaled SINR techniques, as well as their impact on subband beamforming.

The paper is organized as follows. In Section II, we formulate our problem and review existing methods. The minimax MSE and minimax regret beamformers are developed in Section III. In Sections IV and V, we discuss practical considerations and present numerical examples, including a subband beamforming application.

\section{SINR AND MMSE BEAMFORMING}

We denote vectors in $\mathbb{C}^{M}$ by boldface lower case letters and matrices in $\mathbb{C}^{N \times M}$ by boldface upper case letters. The matrix $\mathbf{I}$ is the identity matrix of the appropriate dimension, $(\cdot)^{*}$ is the
Hermitian conjugate of the corresponding matrix, and $\hat{(\cdot)}$ denotes an estimated variable.

\section{A. SINR Beamforming}

One of the main tasks of beamforming is to estimate the source signal amplitude $s(t)$ from a set of array observations

$$
\mathbf{y}(t)=s(t) \mathbf{a}+\mathbf{i}(t)+\mathbf{e}(t), \quad 1 \leq t \leq N .
$$

Here, $\mathbf{y}(t) \in \mathbb{C}^{M}$ is the complex vector of array observations at time $t$ with $M$ being the number of sensors, $s(t)$ is the signal amplitude to be estimated, $\mathbf{a}$ is the known steering vector which depends on the direction of arrival (DOA) of the wavefront plane associated with $s(t), \mathbf{i}(t)$ is the interference, $\mathbf{e}(t)$ is a Gaussian noise vector and $N$ is the number of snapshots [1], [3], [4].

Our goal is to estimate the signal amplitude $s(t)$ from the observations $\mathbf{y}(t)$ using a set of beamformer weights $\mathbf{w}(t)$, where the output of a narrowband beamformer is given by

$$
\hat{s}(t)=\mathbf{w}^{*}(t) \mathbf{y}(t), \quad 1 \leq t \leq N .
$$

Traditionally, the beamformer weights $\mathbf{w}(t)=\mathbf{w}$ are chosen to maximize the SINR

$$
\operatorname{SINR}(\mathbf{w})=\sigma_{s}^{2} \frac{\left|\mathbf{w}^{*} \mathbf{a}\right|^{2}}{\mathbf{w}^{*} \mathbf{R} \mathbf{w}}
$$

where $\mathbf{R}=E\left\{(\mathbf{i}+\mathbf{e})(\mathbf{i}+\mathbf{e})^{*}\right\}$ is the interference+noise covariance matrix, and $\sigma_{s}^{2}$ is the signal power given by $\sigma_{s}^{2}=|s(t)|^{2}$ in the deterministic case, and $\sigma_{s}^{2}=E\left\{|s(t)|^{2}\right\}$ when $s(t)$ is a zero-mean stationary random process.

In practice, the covariance matrix $\mathbf{R}$ is often not available, and is therefore replaced by an estimate. The simplest approach is to use the sample covariance

$$
\widehat{\mathbf{R}}_{s m}=\frac{1}{N} \sum_{t=1}^{N} \mathbf{y}(t) \mathbf{y}(t)^{*}
$$

leading to the Capon beamformer [25], [27]. An alternative choice is the diagonal loading estimate given by

$$
\widehat{\mathbf{R}}_{d l}=\widehat{\mathbf{R}}_{s m}+\xi \mathbf{I}=\frac{1}{N} \sum_{t=1}^{N} \mathbf{y}(t) \mathbf{y}(t)^{*}+\xi \mathbf{I}
$$

for some loading factor $\xi$, resulting in the loaded Capon beamformer [5], [6]. A common choice for $\xi$ is $\xi \approx 10 \sigma^{2}$, where $\sigma^{2}$ is the noise power in a single sensor. Another popular strategy is the eigenspace beamformer [7], in which the inverse covariance matrix is estimated as

$$
\left(\widehat{\mathbf{R}}_{\text {eig }}\right)^{-1}=\left(\widehat{\mathbf{R}}_{s m}\right)^{-1} \mathbf{P}_{s}
$$

where $\mathbf{P}_{s}$ is the orthogonal projection onto the signal subspace.

\section{B. MSE Beamforming}

Since $\operatorname{SINR}(\mathbf{w})=\operatorname{SINR}(\alpha \mathbf{w})$ for any choice of $\alpha$, the weight vector maximizing the SINR is specified up to a constant. Although scaling the beamformer weights will not affect the SINR, it can have an impact on other performance measures. In applications in which the beamformer is used to estimate the 
signal waveform $s(t)$, scaling becomes important, particularly in the context of subband beamforming.

A popular design strategy for determining $\alpha$ is to require that $\mathbf{w}^{*} \mathbf{a}=1$, leading to the MVDR beamformer

$$
\mathbf{w}_{\mathrm{MVDR}}=\frac{1}{\mathbf{a}^{*} \mathbf{R}^{-1} \mathbf{a}} \mathbf{R}^{-1} \mathbf{a}
$$

This beamformer can alternatively be obtained as the solution to

$$
\min _{\mathbf{w}} \mathbf{w}^{*} \mathbf{R} \mathbf{w} \quad \text { subject to } \quad \mathbf{w}^{*} \mathbf{a}=1
$$

which, as we show below, has the interpretation of minimizing the MSE subject to the constraint that the beamformer is unbiased. Although this approach has several optimality properties, it does not necessarily result in a good signal estimate. Instead, we may try to choose $\alpha$ to directly minimize the MSE without requiring an unbiased output.

Assuming $s(t)=s$ is deterministic, where for brevity we omitted the index $t$, the MSE between $s$ and its estimate $\hat{s}$ is

$$
\begin{aligned}
E\left\{|\hat{s}-s|^{2}\right\} & =V(\hat{s})+|B(\hat{s})|^{2} \\
& =\mathbf{w}^{*} \mathbf{R} \mathbf{w}+|s|^{2}\left|1-\mathbf{w}^{*} \mathbf{a}\right|^{2}
\end{aligned}
$$

where $V(\hat{s})=E\left\{|\hat{s}-E\{\hat{s}\}|^{2}\right\}$ is the variance of $\hat{s}$ and $B(\hat{s})=$ $E\{\hat{s}\}-s$ is its bias. When $s$ is a zero-mean random variable with variance $\sigma_{s}^{2}$, the term $|s|^{2}$ is replaced by $\sigma_{s}^{2}$. For concreteness, in the rest of the discussion we assume the deterministic model; however, all the results are valid in the stochastic setting where $|s|^{2}$ is replaced by $\sigma_{s}^{2}$.

Minimizing (9) with respect to w leads to the MMSE beamformer

$$
\mathbf{w}(s)=|s|^{2}\left(\mathbf{R}+|s|^{2} \mathbf{a a}^{*}\right)^{-1} \mathbf{a}=\beta(s) \mathbf{w}_{\mathrm{MVDR}}
$$

where the last equality follows from applying the Matrix Inversion Lemma, and we defined the signal-dependent constant

$$
\beta(s)=\frac{|s|^{2} \mathbf{a}^{*} \mathbf{R}^{-1} \mathbf{a}}{1+|s|^{2} \mathbf{a}^{*} \mathbf{R}^{-1} \mathbf{a}} .
$$

The scaling $\beta(s)$ satisfies $0 \leq \beta(s)<1$, and is monotonically increasing in $|s|^{2}$, so that $\|\mathbf{w}(s)\|<\left\|\mathbf{w}_{\text {MVDR }}\right\|$ for all $s$. Substituting $\mathbf{w}(s)$ back into (9), the minimal attainable MSE, which we denote by $\mathrm{MSEB}_{\mathrm{OPT}}$, is given by

$$
\mathrm{MSE}_{\mathrm{OPT}}=\frac{|s|^{2}}{1+|s|^{2} \mathbf{a}^{*} \mathbf{R}^{-1} \mathbf{a}} .
$$

The MSE of the MVDR beamformer follows from (9) as

$$
\mathrm{MSE}_{\mathrm{MVDR}}=\frac{1}{\mathbf{a}^{*} \mathbf{R}^{-1} \mathbf{a}} \text {. }
$$

Comparing (12) with (13) shows that MSEB ${ }_{\mathrm{OPT}}<$ MSEB $_{M V D R}$ for all $|s|>0$, so that the MMSE approach results in a smaller MSE than the MVDR method with the same SINR.

In practice, we cannot typically implement the MMSE beamformer of (10) since it depends on $|s|$, which is usually unknown. The problem stems from the fact that the bias, and therefore the
MSE of (9) is a function of $|s|$. One approach to eliminating this dependency is to force the bias to 0 and then minimize the MSE, which leads to the problem (8). However, this does not guarantee a small MSE, so that on average the resulting estimate $\hat{s}$ may be far from $s$. Indeed, it is well known that unbiased estimators may often lead to large MSE values.

An alternative strategy is to take advantage of knowledge on $|s|$ and then design a robust beamformer whose MSE is reasonably small across all possible signal values. Here we consider bounds on $|s|$ of the form

$$
L \leq|s| \leq U
$$

which can be available, for example, when the type of source and possible distances from the array are known, as can happen in wireless communications and underwater source localization. Given an uncertainty set of the form (14), we adapt the ideas of [23] and [22] to our context and develop two classes of beamformers that minimize a worst-case MSE measure.

When $L$ and $U$ are not known in advance, we can readily estimate them from the data, as we elaborate on further in Sections IV and V. The situation is similar to the MVDR-based beamformers: In developing these methods it is assumed that the interference+noise covariance matrix $\mathbf{R}$ is known; however, in applications, this matrix is typically estimated from the data. Similarly, our development of the minimax robust beamformers will assume that the bounds $L$ and $U$ and the covariance $\mathbf{R}$ are given. As we show, even when the values are estimated, this methodology leads to improved performance over scaled MVDR based methods that account for the MSE using a scaling optimized in terms of MSE.

\section{RoBUST MSE-BASED BEAMFORMING}

\section{A. Minimax MSE Beamforming}

The first approach we consider is to minimize the worst-case MSE over the set (14). Thus, we seek the beamformer $\mathbf{w}_{\mathrm{MXM}}$ that is the solution to

$$
\begin{aligned}
\min _{\mathbf{w}} \max _{L \leq|s| \leq U} E\left\{|\hat{s}-s|^{2}\right\} \\
\quad=\min _{\mathbf{w}} \max _{L \leq|s| \leq U}\left\{\mathbf{w}^{*} \mathbf{R} \mathbf{w}+|s|^{2}\left|1-\mathbf{w}^{*} \mathbf{a}\right|^{2}\right\}
\end{aligned}
$$

Clearly, the inner maximization is obtained at $|s|=U$ so that our problem reduces to

$$
\min _{\mathbf{w}}\left\{\mathbf{w}^{*} \mathbf{R w}+U^{2}\left|1-\mathbf{w}^{*} \mathbf{a}\right|^{2}\right\}
$$

which is equal to the MSE of (9) with $|s|^{2}=U$. Therefore, $\mathbf{w}_{\mathrm{MXM}}$ is an MMSE beamformer (10) matched to $|s|=U$,

$$
\mathbf{w}_{\mathrm{MXM}}=\frac{U^{2}}{1+U^{2} \mathbf{a}^{*} \mathbf{R}^{-1} \mathbf{a}} \mathbf{R}^{-1} \mathbf{a}=\beta_{\mathrm{MXM}} \mathbf{w}_{\mathrm{MVDR}}
$$

where

$$
\beta_{\mathrm{MXM}}=\frac{U^{2} \mathbf{a}^{*} \mathbf{R}^{-1} \mathbf{a}}{1+U^{2} \mathbf{a}^{*} \mathbf{R}^{-1} \mathbf{a}} .
$$


The resulting MSE is

$$
\operatorname{MSE}_{\mathrm{MXM}}=\frac{U^{4} \mathbf{a}^{*} \mathbf{R}^{-1} \mathbf{a}+|s|^{2}}{\left(1+U^{2} \mathbf{a}^{*} \mathbf{R}^{-1} \mathbf{a}\right)^{2}}
$$

Since we assume that $|s|^{2} \leq U^{2}$,

$$
\mathrm{MSE}_{\mathrm{MXM}} \leq \frac{U^{2}}{1+U^{2} \mathbf{a}^{*} \mathbf{R}^{-1} \mathbf{a}}<\frac{1}{\mathbf{a}^{*} \mathbf{R}^{-1} \mathbf{a}}=\mathrm{MSE}_{\mathrm{MVDR}}
$$

so that for any finite choice of $U$, the minimax MSE approach has smaller MSE than the MVDR method. A surprising result, which follows from [21], is that the MSE superiority of the minimax method holds for any choice of $s$ even when $U$ is not known but estimated from the data as $U=\left|\mathbf{w}_{\text {MVDR }}^{*} \mathbf{y}\right|$. Thus, using the MVDR approach to estimate the signal amplitude and then plugging that into the minimax MSE beamformer is guaranteed to improve the MSE performance over the MVDR method.

The theoretical MSE superiority of the minimax technique is true only if $\mathbf{R}$ is known. As we demonstrate in the simulations in Section V, when the MVDR method is used together with an ad hoc signal power estimate and an estimated $\mathbf{R}$, the performance degrades significantly. If, on the other hand, we treat the value of $U$ as a loose bound on the power, as suggested by our formulation, then estimating $U$ and using the minimax MSE approach can improve the performance over other beamformers.

\section{B. Minimax Regret Beamforming}

In some practical applications, particularly when a lower bound on $|s|$ is known, the minimax MSE approach may be overconservative since it replaces $|s|$ by its largest value. To overcome this possible limitation, we now develop a minimax regret beamformer whose performance is as close as possible to that of the MMSE beamformer that knows $|s|$, for all $L \leq|s| \leq U$.

The regret $\mathcal{R}(s, \mathbf{w})$ is defined as the difference between the MSE using a fixed beamformer $\hat{s}=\mathbf{w}^{*} \mathbf{y}$ and the optimal MSE attainable with an estimator of the form $\hat{s}=\mathbf{w}^{*}(s) \mathbf{y}$ when $s$ is known. Using (12)

$$
\begin{aligned}
\mathcal{R}(s, \mathbf{w}) & =E\left\{\left|\mathbf{w}^{*} \mathbf{y}-s\right|^{2}\right\}-\mathrm{MSE}_{\mathrm{OPT}} \\
& =\mathbf{w}^{*} \mathbf{R} \mathbf{w}+|s|^{2}\left|1-\mathbf{w}^{*} \mathbf{a}\right|^{2}-\frac{|s|^{2}}{1+|s|^{2} \mathbf{a}^{*} \mathbf{R}^{-1} \mathbf{a}} .
\end{aligned}
$$

The minimax regret beamformer $\mathbf{w}_{\mathrm{MXR}}$ is then designed to minimize the worst-case regret

$$
\mathbf{w}_{\mathrm{MXR}}=\arg \min _{\mathbf{w}} \max _{L \leq|s| \leq U} \mathcal{R}(s, \mathbf{w})
$$

The solution to (22) can be obtained from the results of [28] in which a similar problem is considered in the context of estimation in linear models, and is incorporated in Theorem 1 below. A direct proof of this theorem is given in the Appendix.
Theorem 1: Let $s$ denote an unknown signal amplitude in the model $\mathbf{y}=s \mathbf{a}+\mathbf{n}$, where $\mathbf{a}$ is a known vector, and $\mathbf{n}$ is a zeromean random vector with covariance $\mathbf{R}$. Then, the solution to the problem

$$
\min _{\hat{s}=\mathbf{w}^{*} \mathbf{y}} \max _{L \leq|s| \leq U}\left\{E\left\{|\hat{s}-s|^{2}\right\}-\min _{\hat{s}=\mathbf{w}^{*}(s) \mathbf{y}} E\left\{|\hat{s}-s|^{2}\right\}\right\}
$$

is

$$
\mathbf{w}_{\mathrm{MXR}}=\beta_{\mathrm{MXR}} \frac{1}{\mathbf{a}^{*} \mathbf{R}^{-1} \mathbf{a}} \mathbf{R}^{-1} \mathbf{a}=\beta_{\mathrm{MXR}} \mathbf{w}_{\mathrm{MVDR}}
$$

where

$$
\beta_{\mathrm{MXR}}=1-\frac{1}{\sqrt{\left(1+L^{2} \mathbf{a}^{*} \mathbf{R}^{-1} \mathbf{a}\right)\left(1+U^{2} \mathbf{a}^{*} \mathbf{R}^{-1} \mathbf{a}\right)}} .
$$

From Theorem 1, we see that the regret beamformer is also a scaled version of the MVDR method, so that it maximizes the SINR. The scaling $\beta_{\mathrm{MXR}}$ satisfies $0 \leq \beta_{\mathrm{MXR}} \leq 1$ and is monotonically increasing in $L$ and $U$. For $U=L$,

$$
\beta_{\mathrm{MXR}}=\frac{U^{2} \mathbf{a}^{*} \mathbf{R}^{-1} \mathbf{a}}{1+U^{2} \mathbf{a}^{*} \mathbf{R}^{-1} \mathbf{a}}=\beta_{\mathrm{MXM}}
$$

in which case $\mathbf{w}_{\mathrm{MXR}}$ is equal to the minimax MSE beamformer of (19). For $L<U,\left\|\mathbf{w}_{\mathrm{MXR}}\right\|<\left\|\mathbf{w}_{\mathrm{MXM}}\right\|$.

The minimax regret beamformer can also be interpreted as an MMSE beamformer of the form (10), matched to

$$
|s|^{2}=\frac{1}{\gamma}\left(\sqrt{\left(1+L^{2} \gamma\right)\left(1+U^{2} \gamma\right)}-1\right)
$$

where for brevity we denoted

$$
\gamma=\mathbf{a}^{*} \mathbf{R}^{-1} \mathbf{a}
$$

This follows from substituting $|s|^{2}$ of (25) into (10). Since the regret approach minimizes the MSE for the signal power given by (25), we may view this power as an estimate of the true, unknown power. It is interesting to note that while the minimax MSE estimator is matched to a signal power $U^{2}$, the regret estimator is matched to a signal power $|s|^{2} \leq\left(U^{2}+L^{2}\right) / 2$. This follows from (25) by using the inequality $\sqrt{a b} \leq(a+b) / 2$.

To gain further insight into the estimate of $(25)$, we use the equality

$$
\begin{gathered}
\left(\sqrt{\left(1+L^{2} \gamma\right)\left(1+U^{2} \gamma\right)}-1\right)\left(\sqrt{1+L^{2} \gamma}+\sqrt{1+U^{2} \gamma}\right) \\
=U^{2} \gamma \sqrt{1+L^{2} \gamma}+L^{2} \gamma \sqrt{1+U^{2} \gamma}
\end{gathered}
$$

to express $|s|^{2}$ as a weighted combination of $U^{2}$ and $L^{2}$,

$$
\begin{aligned}
& |s|^{2}=\frac{U^{2} \sqrt{1+L^{2} \mathbf{a}^{*} \mathbf{R}^{-1} \mathbf{a}}+L^{2} \sqrt{1+U^{2} \mathbf{a}^{*} \mathbf{R}^{-1} \mathbf{a}}}{\sqrt{1+L^{2} \mathbf{a}^{*} \mathbf{R}^{-1} \mathbf{a}}+\sqrt{1+U^{2} \mathbf{a}^{*} \mathbf{R}^{-1} \mathbf{a}}} \\
& =\eta U^{2}+(1-\eta) L^{2} \text {. }
\end{aligned}
$$

The weight $\eta$ is given by

$$
\eta=\frac{\sqrt{1+\mu(L)}}{\sqrt{1+\mu(L)}+\sqrt{1+\mu(U)}}
$$




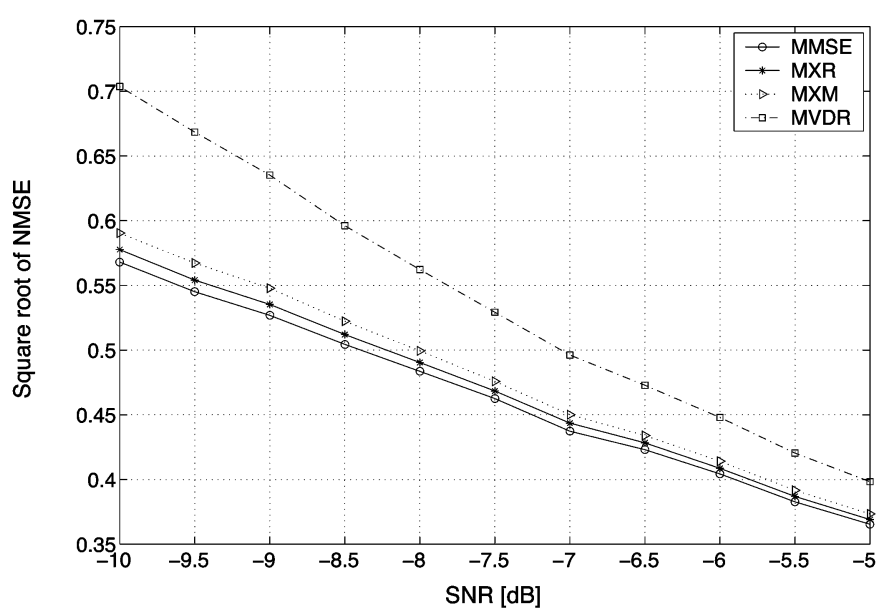

Fig. 1. Square root of the NMSE as a function of SNR using the MMSE, MXR, MXM, and MVDR beamformers in estimating a random process with amplitude uniformly distributed between 3 and 6 .

where $\mu(T)=T^{2} \gamma$ can be viewed as the SNR in the observations when the signal power is $T^{2}$. If $\mu(L) \gg 1$, then

$$
|s|^{2} \approx \sqrt{U^{2} L^{2}}
$$

and the unknown power is estimated as the geometric mean of its bounds. If, on the other hand, $\mu(L) \ll 1$, then

$$
|s|^{2} \approx \frac{1}{2}\left(L^{2}+U^{2}\right)
$$

and the power is estimated as the algebraic mean.

One advantage of the minimax regret approach is that it explicitly accounts for both the upper and the lower bounds on $|s|$, while the minimax MSE method depends only on the upper bound. Therefore, in applications in which both bounds are available, the minimax regret beamformer can lead to better performance. To illustrate this point, in Fig. 1, we compare the MSE of the minimax regret (MXR) and minimax MSE (MXM) methods when estimating a complex random process, temporally white, whose amplitude has a uniform distribution between the values 3 and 6 and its plane-wave has a DOA of $30^{\circ}$, from a uniform linear array (ULA) of $M=20$ omnidirectional sensors spaced half a wavelength apart. The noise e consists of a zero-mean complex Gaussian random vector, spatially and temporally white, with a varying power to obtain the desired SNR, and the interference $\mathbf{i}$ has a DOA of $-30^{\circ}$ and a zero-mean, Gaussian, complex amplitude temporally white with INR of $20 \mathrm{~dB}$. We assume that $\mathbf{R}$ and the bounds $U=6$ and $L=3$ are known. The performance measure we use is the square root of the normalized MSE (NMSE) averaged over $Q$ experiments,

$$
\operatorname{NMSE}_{q}=\sum_{t=1}^{N}\left|\hat{s}_{q}(t)-s_{q}(t)\right|^{2} / \sum_{t=1}^{N}\left|s_{q}(t)\right|^{2}, \quad 1 \leq q \leq Q .
$$

Here, $s_{q}(t)$ and $\hat{s}_{q}(t)$ are the desired signal and its estimate for a given experiment $q$, where we chose $Q=200$. For comparison, we also plot the NMSE of the MVDR strategy and the signaldependent MMSE beamformer, whose performance serves as a bound.
As we expect, the minimax regret beamformer outperforms the minimax MSE and MVDR methods in all the illustrated SNR range, and approaches the MMSE performance. In Section V, we show the advantages of the minimax MSE and minimax regret strategies over several other beamformers for the case where a is known but $\mathbf{R}, U$, and $L$ are estimated from training data containing $s$.

\section{PRACTICAl CONSIDERATIONS}

In our development of the robust MSE beamformers, we assumed that there exist bounds on the magnitude of the signal to be estimated. In some applications, these bounds may be known, for example based on a priori knowledge on the type of the source and its possible range of distances from the array. If no such bounds are available, then we may estimate them from the data using one of the conventional beamformers, denoted $\mathbf{w}_{c}$, by the following procedure.

1) Obtain a rough estimate of $s(t)$ using $\mathbf{w}_{c}: \hat{s}(t)=\mathbf{w}_{c}^{*} \mathbf{y}(t)$.

2) Estimate $U$ and $L$ as $U=(1+\alpha)^{2} \bar{s}$ and $L=(1-\alpha)^{2} \bar{s}$ where $\bar{s}^{2}$ is given by:

a) type $1, \bar{s}^{2}=1 / N \sum_{t=1}^{N} \hat{s}^{2}(t)$ is the average power over the training interval.

b) type $2, \bar{s}^{2}(t)=\hat{s}^{2}(t)$ is the instantaneous signal power estimate, i.e., $U$ and $L$ are time varying.

In the examples below, $\mathbf{w}_{c}$ is chosen as the loaded Capon beamformer and $\alpha$ is set to 4 for the MXR and the MXM beamformers. This value was chosen to give the best performance over a wide range of negative SNR values. We refer to MXM1 and MXM2 for denoting the MXM beamformer with bounds estimated using type $1 \bar{s}^{2}$ and type $2 \bar{s}^{2}$, respectively. We use the same indexing for denoting the MXR beamformer. Since in most applications the true covariance $\mathbf{R}$ is not available, we have to estimate it as well. In the examples below, we use a diagonal loading estimate as in (5) with diagonal load $\xi=10$.

To compare our methods with the SINR-based beamformers, we scaled the corresponding beamformer vectors by $\rho \in \mathbb{C}$. For a fair comparison, we would like to choose $\rho$ to minimize the MSE

$$
E\left\{|s \hat{(t)}-s(t)|^{2}\right\}=|\rho|^{2} \mathbf{w}^{*} \mathbf{R} \mathbf{w}+|s(t)|^{2}\left|1-\rho^{*} \mathbf{w}^{*} \mathbf{a}\right|^{2}
$$

where $\mathbf{w}$ is any beamformer vector. The optimum $\rho$ under this criterion is

$$
\rho=\frac{|s(t)|^{2} \mathbf{w}^{*} \mathbf{a}}{\mathbf{w}^{*}\left(\mathbf{R}+|s(t)|^{2} \mathbf{a a}^{*}\right) \mathbf{w}} .
$$

Note that if $\mathbf{w}=\mathbf{w}_{\mathrm{MVDR}}$, then $\rho=\beta(s)$ in (11). In practice $\mathbf{R}$ and $|s(t)|$ are not known, and therefore need to be estimated. The performance of the normalized SINR-based beamformers depends very much on the way $\rho$ is computed. In particular, the measurement covariance matrix $\left(\mathbf{R}+|s(t)|^{2} \mathbf{a a}^{*}\right)$ can be estimated either with the sample covariance matrix, or by replacing the estimates of $\mathbf{R}$ and $|s(t)|^{2}$. In general, the former approach with type $1|s(t)|^{2}$ estimate in the numerator improves the performance of all SINR-based methods, while the latter degrades it significantly. In our examples below we will use the former scaling approach. 


\section{NUMERICAL EXAMPLES}

To evaluate and compare the performance of our methods with other techniques, we conducted two kinds of examples. We consider the cases for narrowband and wideband $s(t)$. The narrowband signal estimation example is presented in Section V-A, where we made two choices for $s(t)$ : a deterministic complex sinewave, and a zero-mean, temporally white, Gaussian, complex random signal. The wideband signal estimation example is shown in Section V-B, where $s(t)$ was chosen to be a deterministic signal and the estimation is performed using narrowband beamformers with a subband approach.

In both examples, we use a uniform linear array of $M=10$ omnidirectional sensors spaced half a wavelength apart. The signal $s(t)$ is continuously present throughout the training data, and it has a varying amplitude or variance respectively, to obtain the desired SNR in each sensor. Unless otherwise stated, we use a signal DOA equal to $30^{\circ}$ relative to the array normal, a single interference with $\mathrm{INR}=20 \mathrm{~dB}$ and $\mathrm{DOA}=-30^{\circ}$, and equal noise power in each sensor. To illustrate the performance of the beamformers we use the square-root of the NMSE, which is obtained by averaging 500 simulations. In our examples, we focus on low SNR values (important e.g., in sonar) and compare the performance of the proposed methods against six alternative approaches: the Capon beamformer (CAPON) [25], [27], loaded Capon beamformer (L-CAPON) [5], [6], eigenspacebased beamformer (EIG) [7], [8], and three robust beamformers proposed in the literature, which we refer to, respectively, as ROB1 [11], ROB2 [12], and ROB3 [10]. For each method, we tested both the unscaled version and the scaled method, which accounts for the MSE, as described in the previous section. The parameters of each of the existing methods were chosen as suggested in the literature. Namely, for L-CAPON (5) the diagonal loading was set to $\xi=10 \sigma_{e}^{2}$ [10], [11] with $\sigma_{e}^{2}=1$ being the noise power in each sensor. For EIG the number of interferences is set to $n_{i}=1$. For ROB $1 \epsilon=3.5$, for ROB2, $\epsilon=9$, and for ROB3 the upper bound on the steering vector uncertainty was set as $\epsilon=3$ [10]. Note that ROB2 is equal to the L-CAPON beamformer if $\epsilon=0$. In [11], it has been demonstrated that the ROB 1 beamformer vector is equivalent to the ROB3 beamformer; therefore, both are shown as ROB1 in the simulations. Table I summarizes the beamformers analyzed. Note that $\mathbf{P}\{\mathbf{A}\}$ denotes the principal eigenvector of the matrix $\mathbf{A}$ (i.e., the eigenvector associated to the largest eigenvalue of $\mathbf{A}$ ), $\lambda$ is the Lagrange multiplier (see [10] and [11], respectively, for details on its computation), $\alpha_{1}=\left\|\left(\widehat{\mathbf{R}}_{s m}^{-1}+\lambda \mathbf{I}\right)^{-1} \mathbf{a}\right\|$, $\alpha_{2}$ is chosen such that the corresponding beamformer satisfies $\mathbf{w}^{*}\left(\mathbf{a a}^{*}-\epsilon \mathbf{I}\right) \mathbf{w}=1, \alpha_{3}$ is selected such that $\mathbf{w}^{*} \mathbf{R}_{s} \mathbf{w}=1$, and $\beta_{\mathrm{MXR}}$ is defined by (23).

\section{A. Estimating a Complex Sinewave and a Zero-Mean Complex Gaussian Random Process}

In this example, we first choose $s(t)$ in (1) to be a complex sinewave. The noise $\mathbf{e}(t)$ is a zero-mean, Gaussian, complex random vector, temporally and spatially white. The interference is given by $\mathbf{i}(t)=\mathbf{a}_{i} i(t)$ where $i(t)$ is a zero-mean, Gaussian, complex process independent of the noise, and $\mathbf{a}_{i}$ is
TABLE I

BEAMFORMERS USED IN THE NUMERICAL EXAMPLES

\begin{tabular}{|c|c|c|c|}
\hline Beamformer & $\mathbf{w}$ & Parameters & Ref. \\
\hline MXM & $\frac{U^{2}}{1+U^{2} \mathbf{a}^{*} \widehat{\mathbf{R}}_{d l}^{-1} \mathbf{a}} \widehat{\mathbf{R}}_{d l}^{-1} \mathbf{a}$ & $U$ & (17) \\
\hline MXR & $\frac{\beta_{\mathrm{MXR}}}{\mathbf{a}^{*} \widehat{\mathbf{R}}_{d l}^{-1} \mathbf{a}} \widehat{\mathbf{R}}_{d l}^{-1} \mathbf{a}$ & $U, L$ & $(22)$ \\
\hline L-CAPON & $\frac{1}{\mathbf{a}^{*} \widehat{\mathbf{R}}_{d l}^{-1} \mathbf{a}} \widehat{\mathbf{R}}_{d l}^{-1} \mathbf{a}$ & $\xi=10$ & {$[6]$} \\
\hline EIG & $\frac{1}{\mathbf{a}^{*} \widehat{\mathbf{R}}_{e i g}^{-1} \mathbf{a}} \widehat{\mathbf{R}}_{e i g}^{-1} \mathbf{a}$ & $n_{i}=1$ & {$[7]$} \\
\hline ROB1 & $\frac{\alpha_{1}\left(\lambda \widehat{\mathbf{R}}_{s m}+\mathbf{I}\right)^{-1} \mathbf{a}}{\sqrt{M} \mathbf{a}^{*}\left(\lambda \widehat{\mathbf{R}}_{s m}+\mathbf{I}\right)^{-1} \widehat{\mathbf{R}}_{s m}\left(\lambda \widehat{\mathbf{R}}_{s m}+\mathbf{I}\right)^{-1} \mathbf{a}}$ & $\epsilon=3.5$ & {$[11]$} \\
\hline ROB2 & $\alpha_{2} \mathbf{P}\left\{\widehat{\mathbf{R}}_{d l}^{-1}\left(\mathbf{a a}^{*}-\epsilon \mathbf{I}\right)\right\}$ & $\xi=30, \epsilon=9$ & {$[12]$} \\
\hline ROB3 & $\frac{\lambda}{\lambda \mathbf{a}^{*}\left(\widehat{\mathbf{R}}_{s m}+\lambda \epsilon^{2} \mathbf{I}\right)^{-1} \mathbf{a}-1}\left(\widehat{\mathbf{R}}_{s m}+\lambda \epsilon^{2} \mathbf{I}\right)^{-1} \mathbf{a}$ & $\epsilon=3$ & {$[10]$} \\
\hline
\end{tabular}

the interference steering vector. Here, unless otherwise stated, we use $N=50$. The square root of the NMSE as a function of the SNR, the length of training data, the difference between signal and interference DOAs, and the SIR are depicted in Fig. 2(a)-(d).

In Fig. 2(a), we plot the square root of the NMSE as a function of the SNR. In this case, we use a type 2 estimator of $|s(t)|$ for our algorithms, so we show MXR2 and MXM2. Since in all the scenarios considered in this example the NMSE of the CAPON beamformer was out of the illustrated scales, we do not plot the performance of this method. Also, it is straightforward to show that L-CAPON and L-CAPON scaled $_{\text {are equiv- }}$ alent so we just illustrate the former one. From Fig. 2(a) it can be seen that the MXR2 beamformer has the best performance for SNR values between -8 to $2 \mathrm{~dB}$. In Fig. 2(b), we plot the square root of the NMSE as a function of the number of training data with $\mathrm{SNR}=-5 \mathrm{~dB}$. It can be seen that the difference in performance between our methods and the $\mathrm{EIG}_{\text {scaled }}$ and ROB2 ${ }_{\text {scaled }}$ beamformers increases noticeably as the number of samples increases. The performance of the proposed methods as a function of the difference between the signal and interference DOAs is illustrated in Fig. 2(c). The NMSE of all the methods remains almost constant for DOA differences between $10^{\circ}$ and $90^{\circ}$ but deteriorates for DOA differences close to $0^{\circ}$. In this case, the MXR2 and MXM2 outperform the others methods for DOA differences larger than $6^{\circ}$. Fig. 2(d) illustrates the performance as a function of the signal-to-interference-ratio (SIR) for an SNR of $-5 \mathrm{~dB}$. The minimax approaches improve their performance as the SIR increases, however, this observation is not very clear for the $\mathrm{EIG}_{\text {scaled }}$ and ROB2 $2_{\text {scaled }}$ beamformers.

Our second choice for $s(t)$ is a zero-mean, temporally white, Gaussian, complex random signal. The square root of the NMSE as a function of the SNR, the number of training data, the difference between signal and interference DOAs, and the SIR are depicted in Figs. 2(e)-(h), respectively. In this case, we only use MXM1 and MXR1 beamformers since the signal variance 


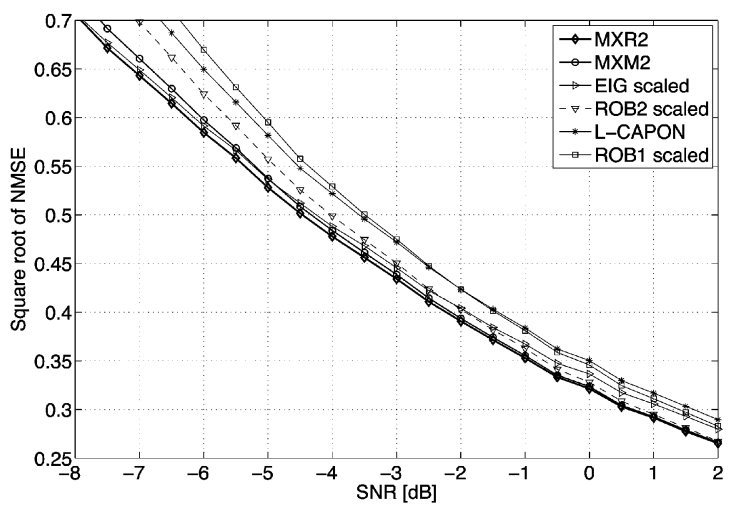

(a)

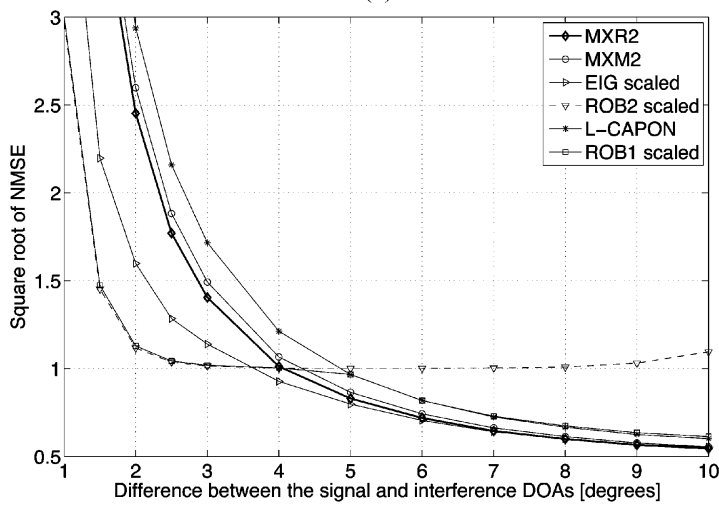

(c)



(e)

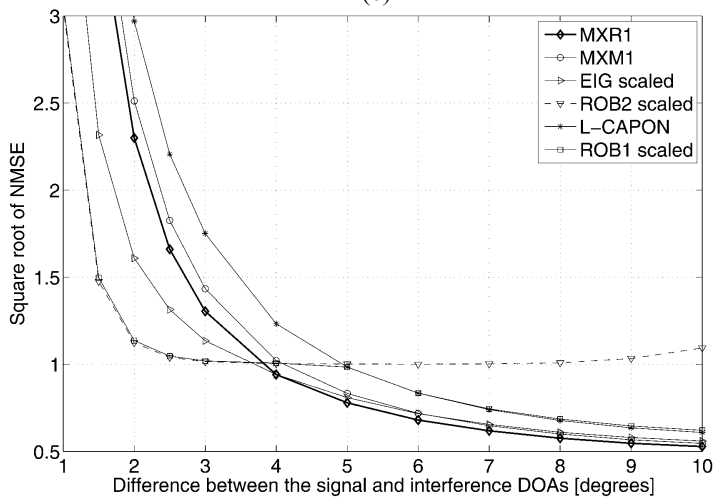

(g)

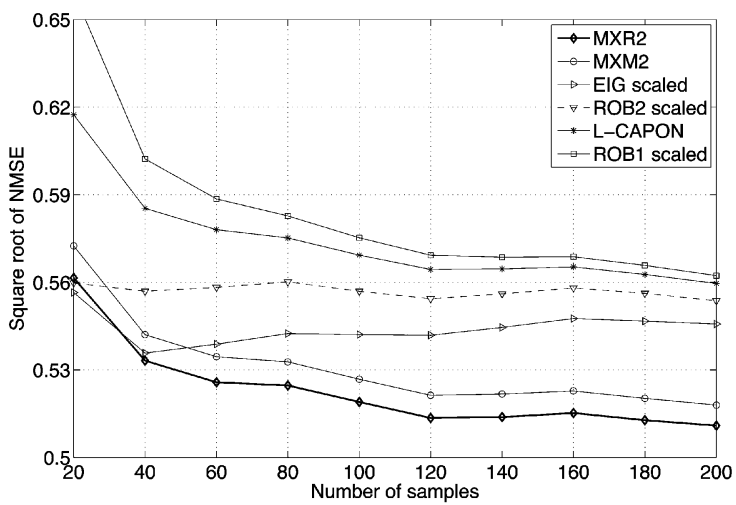

(b)

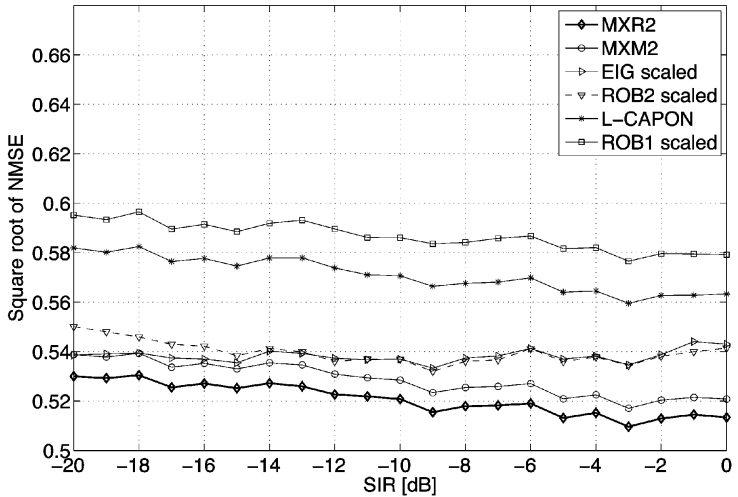

(d)

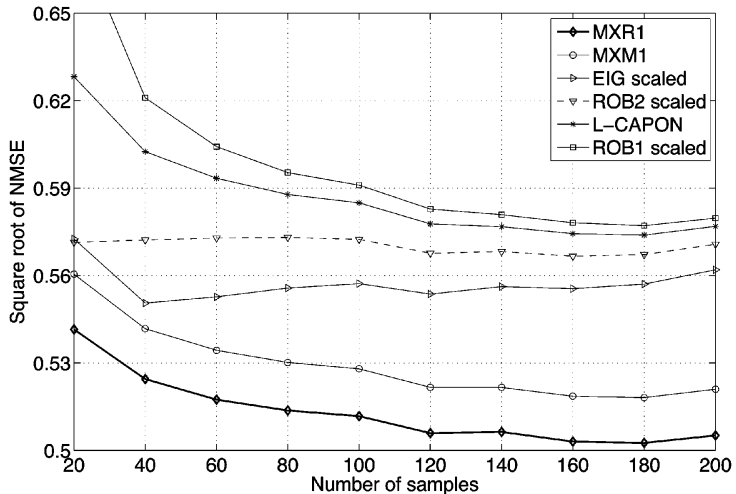

(f)

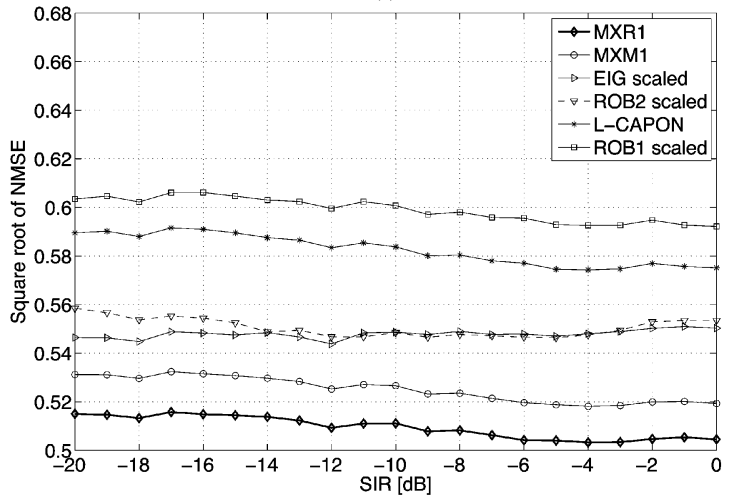

(h)

Fig. 2. Square root of the NMSE as a function of SNR, length of training data, the difference between the signal and interference DOAs, and SIR, when estimating (a)-(d) a complex sinewave and (e)-(h) a zero-mean complex Gaussian random signal with DOA $=30^{\circ}$, using the MXR1, MXM1, EIG scaled, $\mathrm{L}_{-} \mathrm{CAPON}$, $\mathrm{ROB} 1_{\text {scaled }}$, and ROB $2_{\text {scaled }}$ beamformers.

is constant. It can be seen in Fig. 2(e) that the MXR1 has the best performance for SNR values between -8 to $0 \mathrm{~dB}$. The per- formance conclusions from Fig. 2(f)-(h) are similar to the case of a deterministic sinewave. 


\section{B. Estimating a Deterministic Wideband Signal Using a Subband Beamforming Scheme}

We now apply narrowband beamformers in a subband context to estimate a transmitted wideband signal $s(t)$ with bandwidth $B$ and carrier frequency $f_{c}$.

Let

$y_{m}(t)=s\left(t-\tau_{m}(\theta) / T\right) \exp \left(-j 2 \pi f_{c} \tau_{m}(\theta)\right)+i_{m}(t)+e_{m}(t)$

be the complex baseband received signal at the $m$ th sensor [4], where $s(t)=A \operatorname{sinc}^{2}\left(f_{b}(t-50)\right) \cos \left(2 \pi f_{b}(t-50)\right)$, with $f_{b}=f_{c} / 8 \mathrm{~Hz}, f_{c}=1 \mathrm{GHz}$ so that signal bandwidth is $B=4 f_{b} \mathrm{~Hz}$. The signal time delay is given by $\tau_{m}(\theta)=(m-1) \sin (\theta) /\left(2\left(f_{c}+B / 2\right)\right)$ where $\theta$ is the signal DOA, $T$ is the sampling period. The noise components $e_{m}(t)$ are zero-mean, Gaussian, complex random process, spatially white, and $i_{m}(t)=\exp \left(-j, 2, \pi f_{c} \tau_{m}\left(\theta_{i}\right)\right) i\left(t-\tau_{m}\left(\theta_{i}\right) / T\right)$, where $i(t)$ is a zero-mean, Gaussian, complex process, and $\theta_{i}$ is the interference DOA. Both $e_{m}(t)$ and $i(t)$ have a flat spectrum in $\left[-f_{s} / 2, f_{s} / 2\right]$, where $f_{s}=3 f_{c} / 4 \mathrm{~Hz}$ is the sampling frequency.

Since $s(t)$ is wideband, it varies fast compared to the propagation time of the signal wave across the array. Therefore, we cannot model the time delay of the signal as a simple phase shift of the carrier frequency, as in (1). The classical method of tapped delay line [29], [30] overcomes this difficulty by combining spatial filtering with temporal filtering, which makes the response of the array the same across different frequencies. However, this approach has slow convergence and requires a large number of computations [31]. A computationally cheaper strategy is subband beamforming [15]-[20], [31], in which the wideband signal is decomposed into a number of frequency bands by means of a filter bank. These subbands have a reduced bandwidth allowing the application of narrowband beamformers. The summation over the individual beamformers outputs in each subband yields the wideband signal estimate.

Here, we apply the subband method of [17] to design analysis and synthesis FIR uniform DFT-filter banks using unconstrained quadratic optimization. This approach minimizes aliasing in the subband signals as well as magnitude, phase, and aliasing distortion in the reconstructed output signal (see [17] for details). In this scheme, the parameters that need to be set are the number of subbands $K$, the analysis and synthesis filter lengths $n_{h}$ and $n_{g}$, respectively, the decimation factor $D$, the passband cut-off frequency $\omega_{p}$, the delay of the analysis filter bank $\tau_{H}$, and the total subband filtering delay $\tau_{T}$. Fig. 3 illustrates the subband beamformer implemented in this example. This scheme splits the set of array observations $y_{m}(t)$ into $K$ subbands using bandpass filters $h_{k}(t)$, $k=1, \ldots, K$, where the subband bandwidth is $B_{K}=f_{s} / K$ with $K$ chosen such that $B_{K} /\left(2\left(f_{c}+B / 2\right)\right) \ll 1$. Each subband is then processed at a lower sampling rate, using a decimator with rate $D$, where we assume that $N / D$ is an integer. Let $y_{m}^{k}(n)=\sum_{i=1}^{n_{h}} h_{k}(i) y_{m}(n D-i), n=1, \ldots, N / D$ be the downsampled filtered version of $y_{m}(t)$. Denoting $\mathbf{y}^{k}(n)=\left[y_{1}^{k}(n), \ldots, y_{m}^{k}(n), \ldots, y_{M}^{k}(n)\right]^{T}$ and using the fact that $y_{m}^{k}(n)$ is a narrowband signal, we obtain the vector model

$$
\mathbf{y}^{k}(n)=\mathbf{a}_{k} s^{k}(n)+\mathbf{i}^{k}(n)+\mathbf{e}^{k}(n)
$$

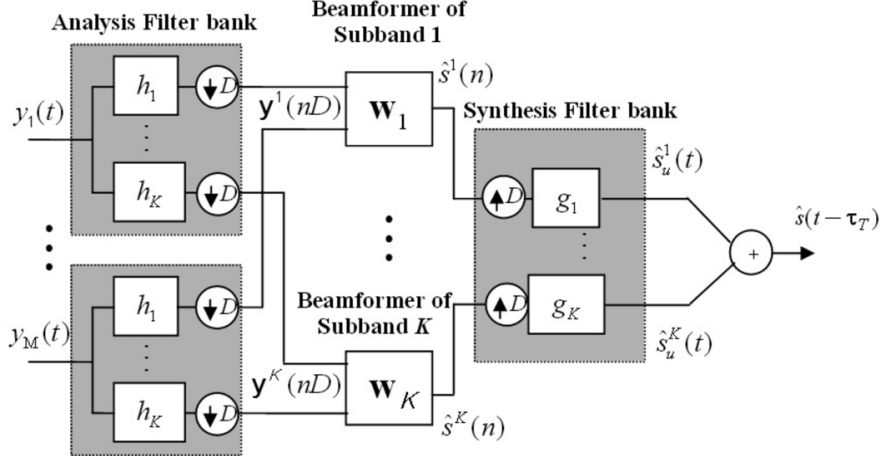

Fig. 3. Subband beamformer scheme implemented for wideband signal waveform estimation.

where $s^{k}(n), \mathbf{i}^{k}(n)$, and $\mathbf{e}^{k}(n)$ are the downsampled outputs of filtering $s(t), \mathbf{i}(t)$, and $\mathbf{e}(t)$ with $h_{k}(t)$, respectively, and $\mathbf{a}_{k}$ is the steering vector

$$
\mathbf{a}_{k}=\left[1, \ldots, e^{-j 2 \pi\left(f_{k}+f_{c}\right) \tau_{m}(\theta)}, \ldots, e^{-j 2 \pi\left(f_{k}+f_{c}\right) \tau_{M}(\theta)}\right]^{T}
$$

where $f_{k}$ is the $k$ th subband center frequency. Using the model (36), we can now design subband beamformers $\mathbf{w}^{k}(n)$ toestimate $s^{k}(n)$ from $\mathbf{y}^{k}(n)$, where the interference+noise covariance matrix for subband $k$ is

$$
\mathbf{R}_{k}=E\left\{\left(\mathbf{i}^{k}(n)+\mathbf{e}^{k}(n)\right)\left(\mathbf{i}^{k}(n)+\mathbf{e}^{k}(n)\right)^{*}\right\} .
$$

To restore the original sampling rate on $\hat{s}^{k}(n)$, we apply a synthesis filter which first upsamples with rate $D$, and then interpolates with an FIR bandpass filter $g_{k}(t): \hat{s}_{u}^{k}(t)=\sum_{i=1}^{N / D} g_{k}(t-$ $i D) \hat{s}^{k}(i)$. The wide band signal estimate is then given by

$$
\hat{s}(t)=\sum_{k=1}^{K} \hat{s}_{u}^{k}\left(t+\tau_{T}\right)
$$

where $\tau_{T}$ is the delay introduced by the analysis and synthesis filters.

In our simulations, we use $K=128$ subbands and $N=256$. The analysis and synthesis filters have the following characteristics [17]: $n_{h}=128, n_{g}=n_{h}, D=64, \omega_{p}=\pi /(12 K)$, $\left.\tau_{H}=\left\lceil\left(n_{h}-1\right) / 2\right)\right\rceil$, and $\tau_{T}=2 \tau_{H}$.

We first assume that the subband array covariance matrix $\mathbf{R}_{k}$, and the subband steering vector $\mathbf{a}_{k}$ are known for $k=1, \ldots, K$. The MMSE beamformer uses the knowledge of $\left|s_{k}(t)\right|^{2}$ for $k=1, \ldots, K$, and in the case of the MXM and MXR beamformers we use $U_{k}=4 P_{w}\left(s^{k}\right)$ and $L_{k}=0.5 P_{w}\left(s^{k}\right)$, where $P_{w}\left(s^{k}\right)$ is the average signal power in subband $k$. Fig. 4(a) illustrates the square-root of the NMSE as a function of SNR using the MMSE, MXR, MXM, and MVDR beamformers. As in the narrowband example, we observe that for low SNR values the performance of the MMSE and robust MSE beamformers are better than the MVDR method. As the SNR increases the behavior of all beamformers tends to be similar. In Fig. 4(b), we show one realization of the absolute value of the signal waveform estimates using the same beamformers for SNR $=-1 \mathrm{~dB}$. It can be seen that the waveform estimates using the MSE criterion are closer to the true waveform $s(t)$ than the one obtained using MVDR. In implementing the MVDR beamformer, the 




(a)
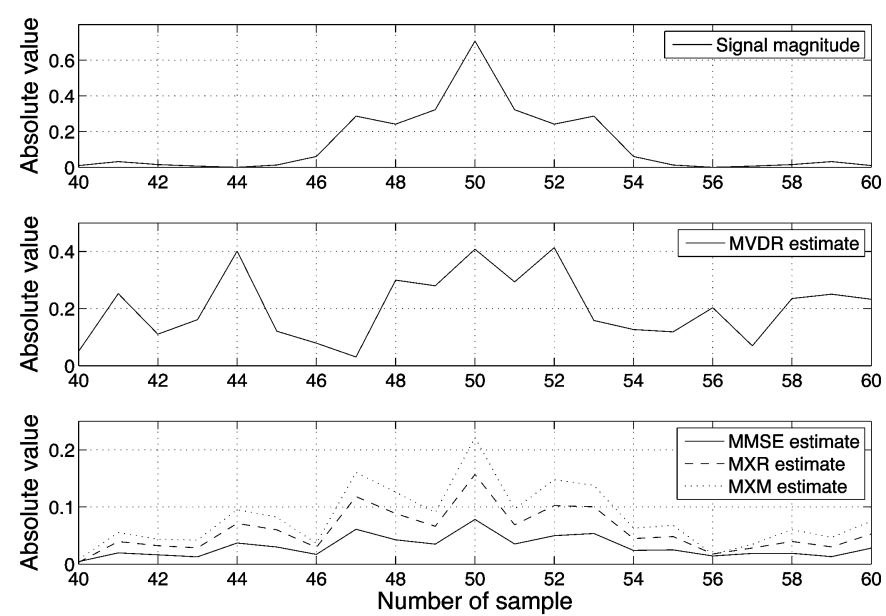

(b)

Fig. 4. Performance of estimating a deterministic wideband signal using a subband beamformer scheme in the presence of an interference with INR $=20 \mathrm{~dB}$ and DOA $=-30^{\circ}$. We assume that $\left|s_{k}\right|^{2}, U_{k}, L_{k}, k=1, \ldots, K$ and the covariance matrices $\mathbf{R}_{k}$ are known. (a) Square root of the NMSE as a function of SNR using the MMSE, MXR, MXM, and MVDR beamformers. (b) Absolute values of: the true signal, the waveform estimate using the MVDR beamformer, and the waveform estimate using MMSE, MXR, and MXM beamformers.

scaling coefficient was chosen optimally such that the received signal waveform magnitude is not distorted in each subband. However, it can be seen that choosing the scaling function according to the MSE criterion leads to estimates that are closer to the true signal waveform.

Next, we considered a more realistic scenario where the bounds as well as the covariance matrix are estimated from the data. In each subband, we implemented the MXR1 and MXM1 beamformers with the sample covariance matrix estimated using a loading factor $\xi=10 \sigma_{e, k}^{2}$ [10], [11], where

$$
\sigma_{e, k}^{2}=\sigma_{e}^{2} \sum_{i=1}^{n_{h}}\left|h_{k}(i)\right|^{2}
$$

is the noise power in the $k^{\text {th }}$ subband. Similarly as in the first case, we estimate the bounds $U_{k}$ and $L_{k}$ with $\mathbf{w}_{c}$ given by the L-CAPON beamformer with $\xi=10 \sigma_{w, k}^{2}$, and type $2|s(t)|^{2}$ estimate with $\alpha$ set as 4 , for these beamformers, respectively. We choose $N=2048$, so that in each subband we use 32 samples to estimate the sample covariance matrix. Fig. 5 illustrates the square root of the NMSE as a function of SNR using the MXM2, MXR2, L-CAPON, ROB1, and ROB1 $1_{\text {scaled }}$ beamformers. It can be seen that the MXR2 and MXM2 beamformers outperform the SINR-based methods in the SNR range illustrated. Between $-5 \mathrm{~dB}$ and $0 \mathrm{~dB}$, the MXR1 and the ROB1 $1_{\text {scaled }}$ beamformers perform similarly.

\section{SUMMARY}

We developed beamforming methods to estimate a source signal $s(t)$ from sensor array observations, where the goal is to obtain an estimate $\hat{s}(t)$ that is close to $s(t)$. Although standard beamforming approaches are aimed at maximizing the SINR, this does not necessarily guarantee a small MSE, hence on average a signal estimate maximizing the SINR can be far from $s(t)$. To ensure that $\hat{s}(t)$ is close to $s(t)$, we proposed using the more appropriate design criterion of MSE. Since the MSE depends in general on $s(t)$ which is unknown, we suggested

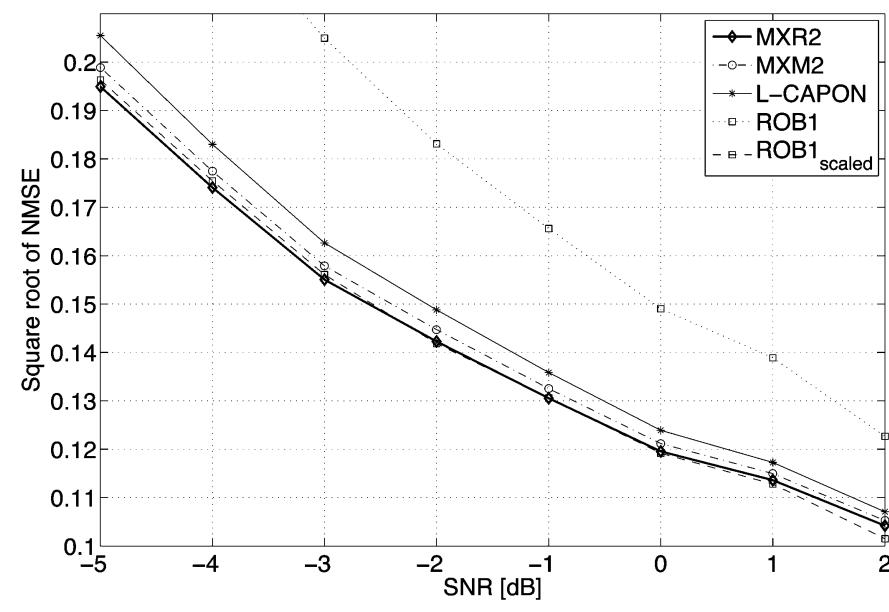

Fig. 5. Square root of the NMSE as a function of SNR using the MXR2,

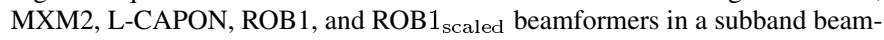
former scheme, to estimate a deterministic wideband signal in the presence of an interference with INR $=20 \mathrm{~dB}$ and $\mathrm{DOA}=-30^{\circ}$.

beamforming strategies that minimize a worst-case measure of MSE assuming bounded signal power. We first considered a minimax MSE approach that minimizes the worst-case MSE. We then proposed a minimax regret method that minimizes the worst-case difference between the MSE using a beamformer ignorant of $|s(t)|$ and the smallest possible MSE attainable when $|s(t)|$ is known. The proposed methods all maximize the SINR but, in general, have better MSE performance than traditional beamformers.

In the numerical examples, we illustrated the advantages of our methods in terms of the MSE, both with respect to SINR based method and scaled SINR techniques which account for the MSE through an optimal scaling factor. We also showed the merits of our techniques in estimating a wideband signal waveform using a subband beamformer approach. In most of the examples shown, the minimax beamformers consistently have the best performance, particularly for negative SNR values. 


\section{APPENDIX A \\ PROOF OF THEOREM 1}

In this Appendix, we prove Theorem 1. Although parts of the proof are similar to those in [26], we repeat the arguments for completeness. The proof can also be found in [13].

To develop a solution to (22), we first consider the inner maximization problem

$$
\begin{aligned}
f(\mathbf{w}) & =\max _{L^{2} \leq x \leq U^{2}}\left\{x\left|1-\mathbf{w}^{*} \mathbf{a}\right|^{2}-\frac{x}{1+x \gamma}\right\} \\
& =\max _{L^{2} \leq x \leq U^{2}} g(x)
\end{aligned}
$$

where we denoted $x=|s|^{2}$ and $g(x)=x\left|1-\mathbf{w}^{*} \mathbf{a}\right|^{2}-x /(1+$ $x \gamma)$. To derive an explicit expression for $f(\mathbf{w})$ we note that the function

$$
h(x)=a x-\frac{b x}{c+d x}
$$

with $b, c, d>0$ is convex in $x \geq 0$ (it is easy to see that $d^{2} h / d x^{2}>0$ for all $x \geq 0$ ). It follows that for fixed $\mathbf{w}, g(x)$ is convex in $x \geq 0$, and consequently the maximum of $g(x)$ over a closed interval is obtained at one of the boundaries. Thus

$$
f(\mathbf{w})=\max \left(g\left(L^{2}\right), g\left(U^{2}\right)\right)
$$

and the problem (22) reduces to

$$
\begin{aligned}
\min _{\mathbf{w}}\left\{\mathbf{w}^{*} \mathbf{R} \mathbf{w}+\max (\right. & L^{2}\left|1-\mathbf{w}^{*} \mathbf{a}\right|^{2}-\frac{L^{2}}{1+L^{2} \gamma}, \\
& \left.\left.U^{2}\left|1-\mathbf{w}^{*} \mathbf{a}\right|^{2}-\frac{U^{2}}{1+U^{2} \gamma}\right)\right\} .
\end{aligned}
$$

We now show that the optimal value of $\mathbf{w}$ has the form

$$
\mathbf{w}=d\left(\mathbf{a}^{*} \mathbf{R}^{-1} \mathbf{a}\right)^{-1} \mathbf{R}^{-1} \mathbf{a}=\frac{d}{\gamma} \mathbf{R}^{-1} \mathbf{a}
$$

for some (possibly complex) $d$. To this end, we first note that the objective in (43) depends on $\mathbf{w}$ only through $\mathbf{w}^{*} \mathbf{a}$ and $\mathbf{w}^{*} \mathbf{R w}$. Now, suppose that we are given a beamformer $\tilde{\mathbf{w}}$, and let

$$
\mathbf{w}=\frac{\mathbf{a}^{*} \tilde{\mathbf{w}}}{\gamma} \mathbf{R}^{-1} \mathbf{a} \text {. }
$$

Then, $\mathbf{w}^{*} \mathbf{a}=\tilde{\mathbf{w}}^{*} \mathbf{a}$ and

$$
\mathbf{w}^{*} \mathbf{R} \mathbf{w}=\frac{\left|\mathbf{a}^{*} \tilde{\mathbf{w}}\right|^{2}}{\gamma^{2}} \mathbf{a}^{*} \mathbf{R}^{-1} \mathbf{a}=\frac{\left|\mathbf{a}^{*} \tilde{\mathbf{w}}\right|^{2}}{\gamma} .
$$

From the Cauchy-Schwarz inequality, for any vector $\mathbf{x}$

$$
\left|\mathbf{a}^{*} \mathbf{x}\right|^{2} \leq \mathbf{a}^{*} \mathbf{R}^{-1} \mathbf{a x}^{*} \mathbf{R x}=\gamma \mathbf{x}^{*} \mathbf{R} \mathbf{x}
$$

Substituting (47) with $\mathbf{x}=\tilde{\mathbf{w}}$ into (46)

$$
\mathbf{w}^{*} \mathbf{R w}=\frac{\left|\mathbf{a}^{*} \tilde{\mathbf{w}}\right|^{2}}{\gamma} \leq \tilde{\mathbf{w}}^{*} \mathbf{R} \tilde{\mathbf{w}}
$$

Since the objective is strictly convex, the solution is unique, so that

$$
\mathbf{w}=\frac{\mathbf{a}^{*} \mathbf{w}}{\gamma} \mathbf{R}^{-1} \mathbf{a}
$$

which implies that $\mathbf{w}$ has the form (44).

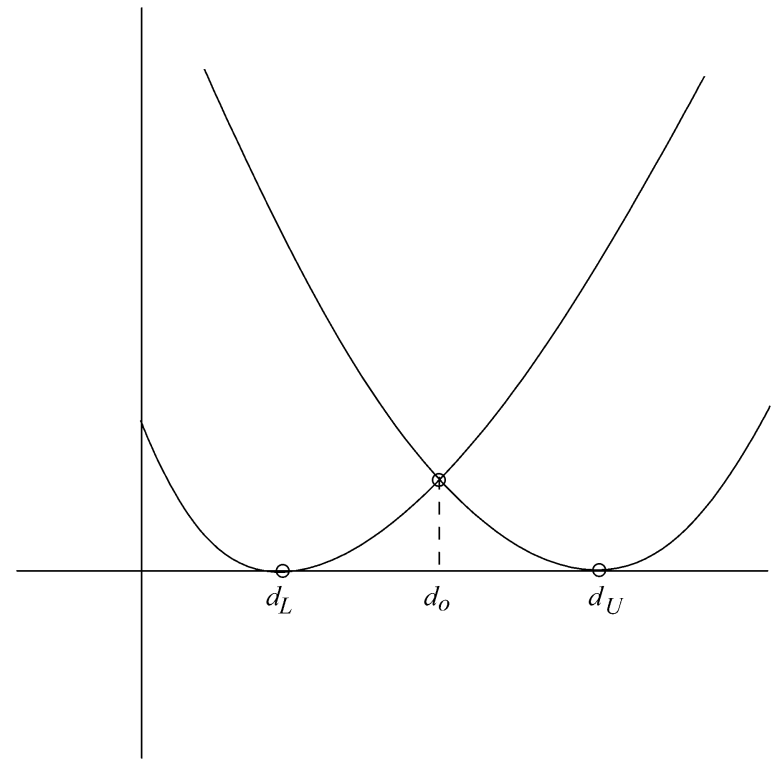

Fig. 6. Illustration of the functions $f_{L}(d)$ and $f_{U}(d)$ of (52).

Combining (44) and (43), our problem reduces to

$$
\begin{aligned}
\min _{d}\left\{\frac{|d|^{2}}{\gamma}+\max \left(L^{2}|1-d|^{2}-\frac{L^{2}}{1+\gamma L^{2}}\right.\right. \\
\left.\left.U^{2}|1-d|^{2}-\frac{U^{2}}{1+\gamma U^{2}}\right)\right\} .
\end{aligned}
$$

Since $d$ is in general complex, we can write $d=|d| e^{j \phi}$ for some $0 \leq \phi \leq 2 \pi$. Using the fact that $|1-d|^{2}=1+|d|^{2}-2 \cos (\phi)$, it is clear that at the optimal solution, $\phi=0$. Therefore, we assume in the sequel that $d \geq 0$. We can then express the problem of (50) as

$$
\min _{t, d}\left\{t: f_{L}(d) \leq t, f_{U}(d) \leq t\right\}
$$

where

$$
\begin{aligned}
& f_{L}(d) \triangleq \frac{d^{2}}{\gamma}+L^{2}(1-d)^{2}-\frac{L^{2}}{1+\gamma L^{2}} \\
& f_{u}(d) \triangleq \frac{d^{2}}{\gamma}+U^{2}(1-d)^{2}-\frac{U^{2}}{1+\gamma U^{2}}
\end{aligned}
$$

To develop a solution to (51) we note that both $f_{L}(d)$ and $f_{U}(d)$ are quadratic functions in $d$, that obtain a minimum at $d_{L}$ and $d_{U}$ respectively, where

$$
d_{L}=\frac{\gamma L^{2}}{1+\gamma L^{2}}, \quad d_{U}=\frac{\gamma U^{2}}{1+\gamma U^{2}}
$$

Therefore, if the functions intersect in the interval $\mathcal{I}=\left[d_{L}, d_{U}\right]$, then the intersection point, denoted $d_{o}$, is the optimal value of $d$, as illustrated in Fig. 6. 
The intersection points of $f_{L}(d)$ and $f_{U}(d)$ are given by

$$
d_{ \pm}=1 \pm \frac{1}{\sqrt{\left(1+\gamma L^{2}\right)\left(1+\gamma U^{2}\right)}} .
$$

Since $d_{+}>1$, clearly $d_{+} \notin \mathcal{I}$. Using the fact that

$$
\frac{1}{1+\gamma U^{2}} \leq \frac{1}{\sqrt{\left(1+\gamma L^{2}\right)\left(1+\gamma U^{2}\right)}} \leq \frac{1}{1+\gamma L^{2}}
$$

we have that $d_{-} \in \mathcal{I}$, and therefore $d_{o}=d_{-}$, completing the proof.

\section{ACKNOWLEDGMENT}

The authors would like to thank the Associate Editor and reviewers for their careful evaluating comments and helpful suggestions that greatly improved the presentation.

\section{REFERENCES}

[1] B. D. V. Veen and K. M. Buckley, "Beamforming: A versatile approach to spatial filtering," IEEE Signal Process. Mag., vol. 5, pp. 4-24, Apr. 1988.

[2] S. Haykin and A. Steinhardt, Adaptive Radar Detection and Estimation. New York: Wiley, 1992.

[3] H. Krim and M. Viberg, "Two decades of array signal processing research: The parametric approach," IEEE Signal Process. Mag., vol. 13, pp. 67-94, Jul. 1996.

[4] H. L. V. Trees, Optimum Array Processing. New York: Wiley, 2002.

[5] B. D. Carlson, "Covariance matrix estimation errors and diagonal loading in adaptive arrays," IEEE Trans. Aerosp. Electron. Syst., vol. 24, pp. 397-401, Jul. 1988.

[6] H. Cox, R. M. Zeskind, and M. M. Owen, "Robust adaptive beamforming," IEEE Trans. Acoust., Speech, Signal Process., vol. ASSP-35, pp. 1365-1376, Oct. 1987.

[7] D. D. Feldman and L. J. Griffiths, "A projection approach for robust adaptive beamforming," IEEE Trans. Signal Process., vol. 42, no. 4, pp. 867-876, Apr. 1994.

[8] N. L. Owsley, "Enhanced minimum variance beamforming," in Underwater Acoustic Data Processing, Y. T. Chan, Ed. Norwell, MA: Kluwer, 1989, pp. 285-292.

[9] K. L. Bell, Y. Ephraim, and H. L. Van Trees, "A Bayesian approach to robust adaptive beamforming," IEEE Trans. Signal Process., vol. 48, no. 2, pp. 386-398, Feb. 2000.

[10] S. A. Vorobyov, A. B. Gershman, and Z.-Q. Luo, "Robust adaptive beamforming using worst-case performance optimization," IEEE Trans. Signal Process., vol. 51, no. 2, pp. 313-324, Feb. 2003.

[11] J. Li, P. Stoica, and Z. Wang, "On robust Capon beamforming and diagonal loading," IEEE Trans. Signal Process., vol. 51, no. 7, pp. 1702-1715, Jul. 2003.

[12] S. Shahbazpanahi, A. B. Gershman, Z.-Q. Luo, and K. M. Wong, "Robust adaptive beamforming for general-rank signal models," IEEE Trans. Signal Process., vol. 51, no. 9, pp. 2257-2269, Sep. 2003.

[13] Y. C. Eldar and A. Nehorai, "Mean-squared error beamforming for signal estimation: A competitive approach," in Robust Adaptive Beamforming, J. Li and P. Stoica, Eds. Hoboken, NJ: Wiley, 2006.

[14] Y. C. Eldar, A. Nehorai, and P. S. La Rosa, "An expected least-squares beamforming approach to signal estimation with steering vector uncertainties," IEEE Signal Process. Lett., pp. 288-291, May 2006.

[15] S. E. Nordholm, I. Claesson, and N. Grbić, "Performance limits in subband beamforming," IEEE Trans. Speech Audio Process., vol. 11, pp. 193-203, May 2003.

[16] N. Grbić, S. E. Nordholm, and A. Cantoni, "Optimal fir subband beamforming for speech enhancement in multipath environments," IEEE Signal Process. Lett., vol. 10, pp. 335-338, Nov. 2003.

[17] J. M. de Haan, N. Grbić, I. Claesson, and S. E. Nordholm, "Filter bank design for subband adaptive microphone arrays," IEEE Trans. Speech Audio Process., vol. 11, pp. 14-23, Jan. 2003.
[18] Y. Chunhua, M. R. Azimi-Sadjadi, J. Wilbur, and G. J. Dobeck, "Underwater target detection using multichannel subband adaptive filtering and high-order correlation schemes," IEEE J. Ocean. Eng., vol. 25, no. 1, pp. 192-205, Jan. 2000.

[19] A. Gilloirc and M. Vetterli, "Adaptive filtering in subbands with critical sampling: Analysis. experiments and applications to acoustic echo cancellation," IEEE Trans. Signal Process., vol. 40, no. 8, pp. 1862-1875, Aug. 1992.

[20] R. Compton, "The relationship between tapped delay-line and FFT processing in adaptive arrays," IEEE Trans. Antennas Propag., vol. 36, no. 1, pp. 15-26, Jan. 1988.

[21] Z. Ben-Haim and Y. C. Eldar, "Blind minimax estimation," Elect. Eng. Dept., Technion-Israel Institute of Technology, Haifa, Israel, CCIT Rep. 550, Aug. 2005

[22] Y. C. Eldar, A. Ben-Tal, and A. Nemirovski, "Robust mean-squared error estimation in the presence of model uncertainties," IEEE Trans. Signal Process., vol. 53, no. 1, pp. 168-181, Jan. 2005.

[23] Y. C. Eldar, A. Ben-Tal, and A. Nemirovski, "Linear minimax regret estimation of deterministic parameters with bounded data uncertainties," IEEE Trans. Signal Process., vol. 52, no. 8, pp. 2177-2188, Aug. 2004.

[24] Y.C. Eldar and N. Merhav, "A competitive minimax approach to robust estimation of random parameters," IEEE Trans. Signal Process., vol. 52, no. 7, pp. 1931-1946, Jul. 2004.

[25] R. T. Lacos, "Data adaptive spectral analysis methods," Geophys., vol. 36, pp. 661-675, Aug. 1971.

[26] Y. C. Eldar, "Robust competitive estimation with signal and noise covariance uncertainties," IEEE Trans. Inf. Theory, vol. 52, pp. 4532-4547, Oct. 2006.

[27] J. Capon, "High resolution frequency-wavenumber spectrum analysis," Proc. IEEE, vol. 57, pp. 1408-1418, Aug. 1969.

[28] Y. C. Eldar and A. Nehorai, "Competitive mean-squared error beamforming," presented at the 12th Annu. Workshop Adaptive Sensor Array Processing, Lexington, MA, Mar. 2004.

[29] W. E. Rodgers and R. T. Compton, "Adaptive array bandwidth with tapped delay-line processing," IEEE Trans. Aerosp. Electron. Syst., vol. AES-15, no. 1, pp. 15-26, Jan. 1979.

[30] J. T. Mayhan, A. J. Simmons, and W. C. Cummings, "Wideband adaptive antenna nulling using tapped delay lines," IEEE Trans. Antennas Propag., vol. AP-29, pp. 923-936, Nov. 1981.

[31] Y. Zhang, S. Weiss, and L. Hanzo, "Subband adaptive antenna array for wideband wireless communications," in Proc. 3rd Int. Conf. Microwave Millimeter Wave Technology (ICCMT), Aug. 2002, pp. 693-696.

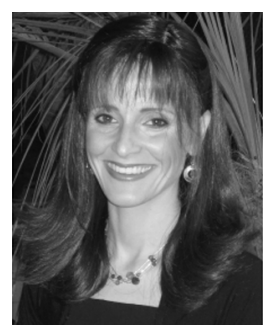

Yonina C. Eldar (S'98-M'02) received the B.Sc degree in physics in 1995 and the B.Sc. degree in electrical engineering in 1996, both from Tel-Aviv University (TAU), Tel-Aviv, Israel, and the Ph.D. degree in electrical engineering and computer science in 2001 from the Massachusetts Institute of Technology (MIT), Cambridge.

From January 2002 to July 2002, she was a Postdoctoral Fellow at the Digital Signal Processing Group at MIT. She is currently an Associate Professor in the Department of Electrical Engineering at the Technion-Israel Institute of Technology, Haifa, Israel. She is also a Research Affiliate with the Research Laboratory of Electronics at MIT. Her research interests are in the general areas of signal processing, statistical signal processing, and computational biology.

Dr. Eldar was in the program for outstanding students at TAU from 1992 to 1996. In 1998, she held the Rosenblith Fellowship for study in Electrical Engineering at MIT, and in 2000, she held an IBM Research Fellowship. From 2002-2005, she was a Horev Fellow of the Leaders in Science and Technology program at the Technion and an Alon Fellow. In 2004, she was awarded the Wolf Foundation Krill Prize for Excellence in Scientific Research, and in 2005 the Andre and Bella Meyer Lectureship, and in 2007, the Henry Taub Prize for Excellence in Research. She is a member of the IEEE Signal Processing Theory and Methods technical committee, an Associate Editor for the IEEE TRANSACTIONS ON SIGNAL PROCESSING and the EURASIP Journal of Signal Processing, and on the Editorial Board of Foundations and Trends in Signal Processing. 


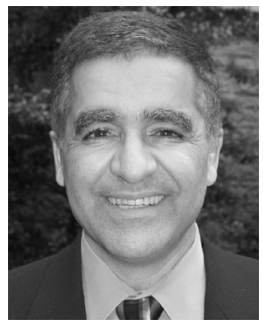

Arye Nehorai (S'80-M'83-SM'90-F'94) received the B.Sc. and M.Sc. degrees in electrical engineering from the Technion, Haifa, Israel, and the Ph.D. degree in electrical engineering from Stanford University, Stanford, CA.

From 1985 to 1995 , he was a faculty member with the Department of Electrical Engineering at Yale University, New Haven, CT. In 1995, he joined as Full Professor the Department of Electrical Engineering and Computer Science at The University of Illinois at Chicago (UIC). From 2000 to 2001, he was Chair of the department's Electrical and Computer Engineering (ECE) Division, which then became a new department. In 2001, he was named University Scholar of the University of Illinois. In 2006, he became Chairman of the Department of Electrical and Systems Engineering at Washington University in St. Louis (WUSTL). He has been the inaugural holder of the Eugene and Martha Lohman Professorship and the Director of the Center for Sensor Signal and Information Processing (CSSIP) at WUSTL since 2006.

Dr. Nehorai was Editor-in-Chief of the IEEE TRANSACTIONS ON SignaL PROCESSING from 2000 to 2002. From 2003 to 2005, he was Vice-President (Publications) of the IEEE Signal Processing Society (SPS), Chair of the Publications Board, member of the Board of Governors, and member of the Executive Committee of the IEEE SPS. From 2003 to 2006, he was the Founding Editor of the special columns on Leadership Reflections in the IEEE
Signal Processing Magazine. He was corecipient of the IEEE SPS 1989 Senior Award for Best Paper with P. Stoica, coauthor of the 2003 Young Author Best Paper Award and corecipient of the 2004 Magazine Paper Award with A. Dogandzic. He was elected Distinguished Lecturer of the IEEE SPS for the term 2004 to 2005 and received the 2006 IEEE SPS Technical Achievement Award. He is the Principal Investigator of the new multidisciplinary university research initiative (MURI) project entitled Adaptive Waveform Diversity for Full Spectral Dominance. He has been a Fellow of the Royal Statistical Society since 1996.

Patricio S. La Rosa received the B.Sc. degree in engineering in 1999, and completed his coursework for the electrical civil-industrial engineering program in 2000, both from the Pontifical Catholic University of Chile (PUC), Chile. He received the M.Sc. degree in electrical engineering (with maximum distinction) from the University of Chile, Chile, in 2003. He is currently working towards the Ph.D. degree under the guidance of Prof. A. Nehorai at the Department of Electrical and Systems Engineering, Washington University in St. Louis, MO.

His research interest are in statistical signal processing and its applications to biomedicine and sensor arrays.

Mr. La Rosa received the John Paul II Foundation scholarship between the years 1995 and 2000 for undergraduate studies in engineering sciences at PUC. 